\title{
Elliptic Systems of Pseudodifferential Equations in the Refined Scale on a Closed Manifold
}

by

\author{
Vladimir A. MIKHAILETS and Aleksandr A. MURACH
}

Presented by Bogdan BOJARSKI

Summary. We study a system of pseudodifferential equations which is elliptic in the Petrovskiı̌ sense on a closed smooth manifold. We prove that the operator generated by the system is a Fredholm operator in a refined two-sided scale of Hilbert function spaces. Elements of this scale are special isotropic spaces of Hörmander-Volevich-Paneah.

1. Introduction. In this article we consider a system of linear pseudodifferential equations elliptic in the Petrovskir sense on a closed smooth manifold. It is known (see $[1,11]$ ) that the operator $A$ corresponding to this system is bounded and Fredholm on appropriate pairs of Sobolev spaces. We investigate this operator on the Hilbert scale of special isotropic HörmanderVolevich- Paneah spaces [9, 10, 23, 25]

$$
H^{s, \varphi}:=H_{2}^{\langle\cdot\rangle^{s} \varphi(\langle\cdot\rangle)}, \quad\langle\xi\rangle:=\left(1+|\xi|^{2}\right)^{1 / 2} .
$$

Here, $s \in \mathbb{R}$ and $\varphi$ is a function parameter slowly varying at $+\infty$ in the Karamata sense. In particular, every standard function

$$
\varphi(t)=(\log t)^{r_{1}}(\log \log t)^{r_{2}} \ldots(\log \ldots \log t)^{r_{n}}, \quad\left\{r_{1}, \ldots, r_{n}\right\} \subset \mathbb{R}, n \in \mathbb{N},
$$

is admissible. This scale was introduced and investigated by the authors in $[15,16]$. It contains the Sobolev scale $\left\{H^{s}\right\} \equiv\left\{H^{s, 1}\right\}$ and is considerably finer. Therefore the collection of spaces (1) is called a refined scale (with respect to the Sobolev scale).

Spaces of the form (1) arise naturally in different spectral problems: convergence of spectral expansions of self-adjoint elliptic operators almost ev-

2000 Mathematics Subject Classification: Primary 35J45; Secondary 46E35.

Key words and phrases: elliptic system, pseudodifferential operator, regularly varying function, scale of spaces, Hörmander spaces, Fredholm property. 
erywhere, or in the norm of $L_{p}$ with $p>2$ or $C$ (see survey [2]); spectral asymptotics of general self-adjoint elliptic operators in a bounded domain; the Weyl formula with a sharp estimate of the remainder (see $[13,14]$ ), and others. They may be expected to be useful in other "fine" questions. Due to their interpolation properties, the spaces $H^{s, \varphi}$ occupy a special position among the spaces of generalized smoothness which are actively investigated and used today (see survey [12], recent articles [8,6] and the bibliography therein).

The main result of this article is Theorem 1 on the boundedness and the Fredholm property of an elliptic operator on the scale (1). The refined local smoothness of a solution of the elliptic system is obtained as an important application (Theorem 4). Also some auxiliary results are given which may be of independent interest. The case of scalar elliptic operators was investigated earlier by the authors in [16-21].

2. The statement of the problem. Let $\Gamma$ be a closed (compact and without boundary) infinitely smooth manifold of dimension $n \geq 1$. We suppose that a certain $C^{\infty}$-density $d x$ is defined on $\Gamma$. By $\mathcal{D}^{\prime}(\Gamma)$ we denote the linear topological space of all distributions on $\Gamma$, that is, $\mathcal{D}^{\prime}(\Gamma)$ is the space antidual to the space $C^{\infty}(\Gamma)$ with respect to the extension of the inner product in $L_{2}(\Gamma, d x)$ by continuity. This extension is denoted by $(f, w)_{\Gamma}$ for $f \in \mathcal{D}^{\prime}(\Gamma), w \in C^{\infty}(\Gamma)$.

We consider a system of linear equations

$$
\sum_{k=1}^{p} A_{j, k} u_{k}=f_{j} \quad \text { on } \Gamma, \quad j=1, \ldots, p .
$$

Here, $p \in \mathbb{N}$ and $A_{j, k}, j, k=1, \ldots, p$, are scalar classical (polyhomogeneous) pseudodifferential operators of arbitrary real orders defined on $\Gamma$ (see e.g. [1, Sec. 2.1]). The complete symbol of the pseudodifferential operator $A_{j, k}$ is an infinitely smooth complex-valued function on the cotangent bundle $T^{*} \Gamma$. The principal symbol of $A_{j, k}$ is a positively homogeneous function of order ord $A_{j, k}$ on every fiber $T_{x}^{*} \Gamma \backslash\{0\}, x \in \Gamma$. The principal symbol is assumed not to be identically zero. We consider equations (2) in the sense of distribution theory, so that $u_{k}, f_{j} \in \mathcal{D}^{\prime}(\Gamma)$. For every $k=1, \ldots, p$ we put

$$
m_{k}:=\max \left\{\operatorname{ord} A_{1, k}, \ldots, \text { ord } A_{p, k}\right\} .
$$

Let us assume the system (2) to be elliptic in the Petrovskiur sense, that is,

$$
\operatorname{det}\left(a_{j, k}^{(0)}(x, \xi)\right)_{j, k=1}^{p} \neq 0 \quad \text { for each } x \in \Gamma, \xi \in T_{x}^{*} \Gamma \backslash\{0\} .
$$

Here, $a_{j, k}^{(0)}(x, \xi)$ is the principal symbol of $A_{j, k}$ if ord $A_{j, k}=m_{k}$, and $a_{j, k}^{(0)}(x, \xi)$ $\equiv 0$ if ord $A_{j, k}<m_{k}$. 
Let us rewrite the system (2) in matrix form: $A u=f$ on $\Gamma$, where $A:=$ $\left(A_{j, k}\right)$ is a square $p \times p$ matrix, and $u=\operatorname{col}\left(u_{1}, \ldots, u_{p}\right), f=\operatorname{col}\left(f_{1}, \ldots, f_{p}\right)$ are function columns. The mapping $u \mapsto A u$ is a continuous linear operator on $\left(\mathcal{D}^{\prime}(\Gamma)\right)^{p}$. We investigate the restrictions of this operator to spaces of the refined scale over $\Gamma$.

3. Auxiliary results. We denote by $\mathcal{M}$ the set of all Borel measurable functions $\varphi:[1,+\infty) \rightarrow(0,+\infty)$ such that $\varphi$ and $1 / \varphi$ are bounded on every compact interval $[1, b]$, and $\varphi$ is slowly varying at $+\infty$ in the Karamata sense, that is,

$$
\lim _{t \rightarrow+\infty} \varphi(\lambda t) / \varphi(t)=1 \quad \text { for each } \lambda>0 .
$$

Let $s \in \mathbb{R}$ and $\varphi \in \mathcal{M}$. We denote by $H^{s, \varphi}\left(\mathbb{R}^{n}\right)$ the set of all tempered distributions $u$ whose Fourier transform $\widehat{u}$ is a locally Lebesgue integrable function in $\mathbb{R}^{n}$ which satisfies the condition

$$
\int\langle\xi\rangle^{2 s} \varphi^{2}(\langle\xi\rangle)|\widehat{u}(\xi)|^{2} d \xi<\infty .
$$

Here, the integral is over $\mathbb{R}^{n}$, and $\langle\xi\rangle:=\left(1+\xi_{1}^{2}+\cdots+\xi_{n}^{2}\right)^{1 / 2}$. In $H^{s, \varphi}\left(\mathbb{R}^{n}\right)$ we define the inner product

$$
(u, v)_{H^{s, \varphi}\left(\mathbb{R}^{n}\right)}:=\int\langle\xi\rangle^{2 s} \varphi^{2}(\langle\xi\rangle) \widehat{u}(\xi) \overline{\widehat{v}(\xi)} d \xi .
$$

The space $H^{s, \varphi}\left(\mathbb{R}^{n}\right)$ is a special isotropic Hilbert case of the spaces introduced by L. Hörmander [9, Sec. 2.2], [10, Sec. 10.1] and L. R. Volevich and B. P. Paneah [25, Sec. 2], [23, Sec. 1.4.2]. In the simplest case where $\varphi(\cdot) \equiv 1$ the space $H^{s, \varphi}\left(\mathbb{R}^{n}\right)$ coincides with the Sobolev space $H^{s}\left(\mathbb{R}^{n}\right)$. It follows from the inclusions

$$
\bigcup_{\varepsilon>0} H^{s+\varepsilon}\left(\mathbb{R}^{n}\right)=: H^{s+}\left(\mathbb{R}^{n}\right) \subset H^{s, \varphi}\left(\mathbb{R}^{n}\right) \subset H^{s-}\left(\mathbb{R}^{n}\right):=\bigcap_{\varepsilon>0} H^{s-\varepsilon}\left(\mathbb{R}^{n}\right)
$$

that in the collection of spaces

$$
\left\{H^{s, \varphi}\left(\mathbb{R}^{n}\right): s \in \mathbb{R}, \varphi \in \mathcal{M}\right\}
$$

the function parameter $\varphi$ defines an additional (subpower) smoothness with respect to the basic (power) $s$-smoothness. In other words, $\varphi$ refines the power smoothness.

The refined scale over the manifold $\Gamma$ is constructed from the scale (3) in the usual way. Let us choose a finite $C^{\infty}$ atlas on $\Gamma$ consisting of local charts $\alpha_{j}: \mathbb{R}^{n} \leftrightarrow U_{j}, j=1, \ldots, r$. Here the open sets $U_{j}$ form a finite covering of $\Gamma$. Let $\chi_{j} \in C^{\infty}(\Gamma), j=1, \ldots, r$, form a partition of unity on $\Gamma$ with $\operatorname{supp} \chi_{j} \subset U_{j}$.

We set

$$
H^{s, \varphi}(\Gamma):=\left\{h \in \mathcal{D}^{\prime}(\Gamma):\left(\chi_{j} h\right) \circ \alpha_{j} \in H^{s, \varphi}\left(\mathbb{R}^{n}\right), j=1, \ldots, r\right\} .
$$


Here, $\left(\chi_{j} h\right) \circ \alpha_{j}$ is the representation of the distribution $\chi_{j} h$ in the local chart $\alpha_{j}$. The inner product in $H^{s, \varphi}(\Gamma)$ is defined by the formula

$$
(f, g)_{H^{s, \varphi}(\Gamma)}:=\sum_{j=1}^{r}\left(\left(\chi_{j} f\right) \circ \alpha_{j},\left(\chi_{j} g\right) \circ \alpha_{j}\right)_{H^{s, \varphi}\left(\mathbb{R}^{n}\right)}
$$

and induces the norm $\|h\|_{s, \varphi}:=(h, h)_{s, \varphi}^{1 / 2}$.

The collection of function spaces

$$
\left\{H^{s, \varphi}(\Gamma): s \in \mathbb{R}, \varphi \in \mathcal{M}\right\}
$$

is called the refined scale over the manifold $\Gamma$. We note the following properties of this scale (see [16, Theorems 3.5, 3.6] or [21, Theorem 3.6]).

Proposition 1. Let $s \in \mathbb{R}$ and $\varphi, \varphi_{1} \in \mathcal{M}$.

(i) $H^{s, \varphi}(\Gamma)$ is a separable Hilbert space, independent (up to equivalent norms) of the choice of the atlas and the partition of unity.

(ii) The imbedding $H^{s, \varphi}(\Gamma) \hookrightarrow \mathcal{D}^{\prime}(\Gamma)$ is continuous.

(iii) $C^{\infty}(\Gamma)$ is dense in $H^{s, \varphi}(\Gamma)$.

(iv) For each $\varepsilon>0$ the following compact and dense imbeddings hold:

$$
H^{s+\varepsilon}(\Gamma) \hookrightarrow H^{s, \varphi}(\Gamma) \hookrightarrow H^{s-\varepsilon}(\Gamma) \quad \text { and } \quad H^{s+\varepsilon, \varphi_{1}}(\Gamma) \hookrightarrow H^{s, \varphi}(\Gamma) .
$$

(v) If $\varphi / \varphi_{1}$ is bounded in a neighborhood of $+\infty$, then we have a continuous dense imbedding $H^{s, \varphi_{1}}(\Gamma) \hookrightarrow H^{s, \varphi}(\Gamma)$. It is compact if $\varphi(t) / \varphi_{1}(t) \rightarrow 0$ as $t \rightarrow+\infty$.

(vi) $H^{s, \varphi}(\Gamma)$ and $H^{-s, 1 / \varphi}(\Gamma)$ are mutually dual (up to equivalent norms) with respect to the sesquilinear form $(\cdot, \cdot)_{\Gamma}$.

In connection with assertion (vi) we note that $\varphi \in \mathcal{M} \Leftrightarrow 1 / \varphi \in \mathcal{M}$. Hence the space $H^{-s, 1 / \varphi}(\Gamma)$ is well defined.

The refined scale (4) admits the following alternative intrinsic description [21, Theorem 3.8]. Let the Riemannian structure on the manifold $\Gamma$ which defines the density $d x$ be given (it is always possible), and let $\Delta_{\Gamma}$ be the Beltrami-Laplace operator on $\Gamma$. For $s \in \mathbb{R}$ and $\varphi \in \mathcal{M}$, we define the function

$$
\varphi_{s}(t):= \begin{cases}t^{s / 2} \varphi\left(t^{1 / 2}\right) & \text { for } t \geq 1, \\ \varphi(1) & \text { for } 0<t<1 .\end{cases}
$$

We consider the operator $\varphi_{s}\left(1-\Delta_{\Gamma}\right)$ in the space $L_{2}(\Gamma, d x)$ as a Borel function of the self-adjoint operator $1-\Delta_{\Gamma}$.

Proposition 2. For any $s \in \mathbb{R}$ and $\varphi \in \mathcal{M}$, the space $H^{s, \varphi}(\Gamma)$ coincides with the completion of $C^{\infty}(\Gamma)$ with respect to the norm $u \mapsto$ $\left\|\varphi_{s}\left(1-\Delta_{\Gamma}\right) u\right\|_{L_{2}(\Gamma, d x)}$ which is equivalent to the norm $\|u\|_{s, \varphi}$. 
The following refinement of the classical Sobolev theorem characterizes separating possibilities of the refined scale [16, Theorem 3.6, Remark 3.3].

Proposition 3. Let $\varphi \in \mathcal{M}$ and let $\varrho \geq 0$ be an integer. The inequality

$$
\int_{1}^{+\infty} \frac{d t}{t \varphi^{2}(t)}<\infty
$$

is equivalent to the continuous imbedding $H^{\varrho+n / 2, \varphi}(\Gamma) \hookrightarrow C^{\varrho}(\Gamma)$. The continuity of this imbedding implies its compactness.

4. The main results. We denote by $A^{+}$the matrix pseudodifferential operator formally adjoint to $A$ with respect to the inner product in $\left(L_{2}(\Gamma, d x)\right)^{p}$. We set

$$
\begin{aligned}
N & :=\left\{u \in\left(C^{\infty}(\Gamma)\right)^{p}: A u=0 \text { on } \Gamma\right\}, \\
N^{+} & :=\left\{v \in\left(C^{\infty}(\Gamma)\right)^{p}: A^{+} v=0 \text { on } \Gamma\right\} .
\end{aligned}
$$

The ellipticity of the system (2) implies that the spaces $N$ and $N^{+}$are finite-dimensional [1, Sec. 3.2].

We recall the following: a bounded linear operator $T: X \rightarrow Y$ between Banach spaces is called Fredholm if its kernel is finite-dimensional and its range $T(X)$ is closed in $Y$ and has finite codimension therein. The Fredholm operator $T$ has finite index ind $T:=\operatorname{dim} \operatorname{ker} T-\operatorname{dim}(Y / T(X))$.

Theorem 1. For any $s \in \mathbb{R}$ and $\varphi \in \mathcal{M}$ the mapping $u \mapsto A u, u \in$ $\left(\mathcal{D}^{\prime}(\Gamma)\right)^{p}$, restricts to a bounded linear operator

$$
A: \prod_{k=1}^{p} H^{s+m_{k}, \varphi}(\Gamma) \rightarrow\left(H^{s, \varphi}(\Gamma)\right)^{p} .
$$

This operator is Fredholm, has kernel $N$ and range

$$
\left\{f \in\left(H^{s, \varphi}(\Gamma)\right)^{p}: \sum_{j=1}^{p}\left(f_{j}, v_{j}\right)_{\Gamma}=0 \forall\left(v_{1}, \ldots, v_{p}\right) \in N^{+}\right\} .
$$

The index of the operator (6) is equal to $\operatorname{dim} N-\operatorname{dim} N^{+}$and is independent of $s$ and $\varphi$.

This theorem specifies, with regard to the refined scale (4), the known propositions on solvability of elliptic systems in the Sobolev scale (see [1, Theorem 3.2.1], [11, Theorems 19.2.1, 19.5.3]).

According to Theorem $1, N^{+}$is the defect subspace of the operator (6). Let us note [3], [1, Sec. $2.3 \mathrm{f}]$ that in the scalar case $(p=1)$ the index of $(6)$ is 0 if $\operatorname{dim} \Gamma \geq 2$. Another sufficient condition for this property is the ellipticity of the system with a parameter on a certain ray $K:=\{\lambda \in \mathbb{C}: \arg \lambda=$ const $\}$ [1, Sec. 4]. 
Theorem 2. For any $s \in \mathbb{R}, \varphi \in \mathcal{M}$ and $\sigma>0$, the following a priori estimate holds true:

$$
\sum_{k=1}^{p}\left\|u_{k}\right\|_{s+m_{k}, \varphi} \leq c\left(\sum_{j=1}^{p}\left\|f_{j}\right\|_{s, \varphi}+\sum_{k=1}^{p}\left\|u_{k}\right\|_{s+m_{k}-\sigma, \varphi}\right) .
$$

Here, the number $c>0$ is independent of the vector-functions $u$ and $f=A u$.

If the spaces $N$ and $N^{+}$are trivial, then the operator (6) is a topological isomorphism. Generally, it is convenient to define the isomorphism with the help of two projectors. We decompose the spaces on which (6) acts into the following direct sums of (closed) subspaces:

$$
\begin{aligned}
\prod_{k=1}^{p} H^{s+m_{k}, \varphi}(\Gamma) & =N \dot{+}\left\{u: \sum_{k=1}^{p}\left(u_{k}, w_{k}\right)_{\Gamma}=0 \forall\left(w_{1}, \ldots, w_{p}\right) \in N\right\}, \\
\left(H^{s, \varphi}(\Gamma)\right)^{p} & =N^{+} \dot{+} A\left(\left(H^{s, \varphi}(\Gamma)\right)^{p}\right) .
\end{aligned}
$$

Denote by $P$ and $P^{+}$respectively the oblique projectors of these spaces onto the second terms in the sums. The projectors are independent of $s$ and $\varphi$.

TheOREM 3. For any $s \in \mathbb{R}$ and $\varphi \in \mathcal{M}$, the restriction of the operator (6) to the subspace $P\left(\prod_{k=1}^{p} H^{s+m_{k}, \varphi}(\Gamma)\right)$ establishes a topological isomorphism

$$
A: P\left(\prod_{k=1}^{p} H^{s+m_{k}, \varphi}(\Gamma)\right) \leftrightarrow P^{+}\left(\left(H^{s, \varphi}(\Gamma)\right)^{p}\right) .
$$

5. The proofs. We start from the following lemma. Denote by $\Psi^{r}(\Gamma)$ the set of all (not necessarily classical) pseudodifferential operators on $\Gamma$ of order $\leq r \in \mathbb{R}$.

Lemma 1. Let $r \in \mathbb{R}$ and $T \in \Psi^{r}(\Gamma)$. Then the restriction of the mapping $u \mapsto T u, u \in \mathcal{D}^{\prime}(\Gamma)$, to the space $H^{\sigma, \varphi}(\Gamma)$ is a bounded operator

$$
T: H^{\sigma, \varphi}(\Gamma) \rightarrow H^{\sigma-r, \varphi}(\Gamma) \quad \text { for every } \sigma \in \mathbb{R}, \varphi \in \mathcal{M} .
$$

This lemma is known for $\varphi \equiv 1$ (the Sobolev scale) [1, Theorem 2.1.2]. We deduce the general case by interpolation with a function parameter. We recall the definition of this interpolation (see [7, 24, 15, 21]).

Let $X:=\left[X_{0}, X_{1}\right]$ be an ordered couple of separable complex Hilbert spaces such that there is a continuous dense imbedding $X_{1} \hookrightarrow X_{0}$. We call such a couple admissible. For the couple $X$ there exists an isometric isomorphism $J: X_{1} \leftrightarrow X_{0}$ such that $J$ is a self-adjoint positive operator on $X_{0}$ with domain $X_{1}$. This operator is uniquely determined by the couple $X$. Let $\psi:(0,+\infty) \rightarrow(0,+\infty)$ be a Borel measurable function. We denote by $\left[X_{0}, X_{1}\right]_{\psi}$ or simply by $X_{\psi}$ the domain of the operator $\psi(J)$ endowed with 
the graph inner product and the corresponding norm:

$$
(u, v)_{X_{\psi}}:=(u, v)_{X_{0}}+(\psi(J) u, \psi(J) v)_{X_{0}}, \quad\|u\|_{X_{\psi}}=(u, u)_{X_{\psi}}^{1 / 2} .
$$

The space $X_{\psi}$ is a separable Hilbert space.

The function $\psi$ is called an interpolation parameter if the following condition is fulfilled for all admissible couples $X=\left[X_{0}, X_{1}\right], Y=\left[Y_{0}, Y_{1}\right]$ of Hilbert spaces and any linear mapping $T$ given on $X_{0}$ : if the restriction of $T$ to $X_{j}$ is a bounded operator $T: X_{j} \rightarrow Y_{j}$ for each $j=0,1$, then the restriction of $T$ to $X_{\psi}$ is also a bounded operator $T: X_{\psi} \rightarrow Y_{\psi}$.

We need the following interpolation property of the refined scale on $\Gamma$ (see [16, Theorem 3.5] or [21, Theorem 3.5]). Let $\varphi \in \mathcal{M}$ and $\varepsilon>0$. We set

$$
\psi(t):= \begin{cases}t^{1 / 2} \varphi\left(t^{1 /(2 \varepsilon)}\right) & \text { for } t \geq 1, \\ \varphi(1) & \text { for } 0<t<1 .\end{cases}
$$

Then $\psi$ is an interpolation parameter and

$$
\left[H^{\sigma-\varepsilon}(\Gamma), H^{\sigma+\varepsilon}(\Gamma)\right]_{\psi}=H^{\sigma, \varphi}(\Gamma) \quad \text { for all } \sigma \in \mathbb{R}
$$

with the equivalence of norms.

Proof of Lemma 1. Fix $\sigma \in \mathbb{R}$ and $\varphi \in \mathcal{M}$. We consider the bounded linear operators on Sobolev spaces:

$$
T: H^{\sigma \mp 1}(\Gamma) \rightarrow H^{\sigma-r \mp 1}(\Gamma) .
$$

According to interpolation formula (10) with $\varepsilon=1$ we get from (11) the existence and boundedness of the operator (9):

$$
\begin{aligned}
T: H^{\sigma, \varphi}(\Gamma)=\left[H^{\sigma-1}(\Gamma), H^{\sigma+1}(\Gamma)\right]_{\psi} & \\
& \rightarrow\left[H^{\sigma-r-1}(\Gamma), H^{\sigma-r+1}(\Gamma)\right]_{\psi}=H^{\sigma-r, \varphi}(\Gamma) .
\end{aligned}
$$

Proof of Theorem 1. Let $s \in \mathbb{R}$ and $\varphi \in \mathcal{M}$. The existence and boundedness of the operator (6) results from the condition $A_{j, k} \in \Psi^{m_{k}}(\Gamma)$ and Lemma 1 for $\sigma:=s+m_{k}$. We deduce the Fredholm property of this operator from the following known fact (see [1, Theorem 3.2.1], [11, Theorem 19.5.3]). Since the system (2) is elliptic in the Petrovski1 sense, there exists a matrix pseudodifferential operator $B=\left(B_{k, j}\right)_{k, j=1}^{p}$ such that $B_{k, j} \in \Psi^{-m_{k}}(\Gamma)$ and

$$
B A=I+T_{1}, \quad A B=I+T_{2},
$$

where $T_{1}, T_{2}$ are matrix pseudodifferential operators whose elements belong to $\Psi^{-\infty}(\Gamma):=\bigcap_{r \in \mathbb{R}} \Psi^{r}(\Gamma)$. As usual, $I$ is the identity operator on $\left(\mathcal{D}^{\prime}(\Gamma)\right)^{p}$. The operator $B$ is called a parametrix for $A$. According to Lemma 1 it is a bounded operator

$$
B:\left(H^{s, \varphi}(\Gamma)\right)^{p} \rightarrow \prod_{k=1}^{p} H^{s+m_{k}, \varphi}(\Gamma) .
$$


Write $T_{1}=\left(T_{1}^{j, k}\right)_{j, k=1}^{p}$ and $T_{2}=\left(T_{2}^{k, j}\right)_{k, j=1}^{p}$. Since $T_{1}^{j, k} \in \Psi^{m_{k}-m_{j}-\varrho}(\Gamma)$ and $T_{2}^{k, j} \in \Psi^{-\varrho}(\Gamma)$ for every $\varrho>0$, by Lemma 1 we have the bounded operators

$$
\begin{array}{ll}
T_{1}: \prod_{k=1}^{p} H^{s+m_{k}, \varphi}(\Gamma) \rightarrow \prod_{j=1}^{p} H^{s+m_{j}+\varrho, \varphi}(\Gamma) & \text { for } \varrho>0, \\
T_{2}:\left(H^{s, \varphi}(\Gamma)\right)^{p} \rightarrow\left(H^{s+\varrho, \varphi}(\Gamma)\right)^{p} & \text { for } \varrho>0 .
\end{array}
$$

In addition, according to Proposition 1(iv) we have compact imbeddings

$$
\begin{aligned}
\prod_{j=1}^{p} H^{s+m_{j}+\varrho, \varphi}(\Gamma) & \hookrightarrow \prod_{k=1}^{p} H^{s+m_{k}, \varphi}(\Gamma) & & \text { for } \varrho>0, \\
\left(H^{s+\varrho, \varphi}(\Gamma)\right)^{p} & \hookrightarrow\left(H^{s, \varphi}(\Gamma)\right)^{p} & & \text { for } \varrho>0 .
\end{aligned}
$$

It follows that $T_{1}$ is compact on $\prod_{j=1}^{p} H^{s+m_{j}, \varphi}(\Gamma)$, and $T_{2}$ is compact on $\left(H^{s, \varphi}(\Gamma)\right)^{p}$.

Thus we have equalities (12), where $B$ is bounded whereas $T_{1}, T_{2}$ are compact on the spaces specified above. It is known (see [1, Theorem 2.3.2], [11, Corollary 19.1.9]) that this is equivalent to the Fredholm property of (6).

Denote by $N_{s, \varphi}$ the kernel of (6) and by $N_{s, \varphi}^{+}$the kernel of the adjoint to (6). Let us show that $N_{s, \varphi}=N$ and $N_{s, \varphi}^{+}=N^{+}$.

Let $u \in N_{s, \varphi}$. In view of (12), (14),

$$
u=B A u-T_{1} u=-T_{1} u \in \prod_{j=1}^{p} H^{s+m_{j}+\varrho, \varphi}(\Gamma) \quad \text { for } \varrho>0 .
$$

Hence $u \in C^{\infty}(\Gamma)$ by Proposition 3. So $N_{s, \varphi} \subseteq N$. The inverse inclusion is evident. Thus we have $N_{s, \varphi}=N$.

Further, according to Proposition 2(vi) we can identify the operators adjoint to (6), (13), (15) with the matrix pseudodifferential operators $A^{+}$, $B^{+}, T_{2}^{+}$formally adjoint to $A, B, T_{2}$ with respect to the inner product in $\left(L_{2}(\Gamma, d x)\right)^{p}$. In this sense $N_{s, \varphi}^{+}$is the kernel of the operator

$$
A^{+}:\left(H^{-s, 1 / \varphi}(\Gamma)\right)^{p} \rightarrow \prod_{k=1}^{p} H^{-s-m_{k}, 1 / \varphi}(\Gamma) .
$$

Therefore $N^{+} \subseteq N_{s, \varphi}^{+} \subset\left(H^{-s, 1 / \varphi}(\Gamma)\right)^{p}$. To prove the inclusion $N_{s, \varphi}^{+} \subseteq N^{+}$, let $f \in N_{s, \varphi}^{+}$. Passing to adjoint operators in the second equality of (12) we can write

$$
f=B^{+} A^{+} f-T_{2}^{+} f=-T_{2}^{+} f .
$$

Since all elements of the matrix $T_{2}^{+}$are in $\Psi^{-\infty}(\Gamma)$, by Lemma 1 we have the bounded operator

$$
T_{2}^{+}:\left(H^{-s, 1 / \varphi}(\Gamma)\right)^{p} \rightarrow\left(H^{-s+\varrho, 1 / \varphi}(\Gamma)\right)^{p} \quad \text { for } \varrho>0 .
$$

Proposition 3 now yields $f \in\left(C^{\infty}(\Gamma)\right)^{p}$. Hence $f \in N$, so that $N_{s, \varphi}^{+} \subseteq N^{+}$. Thus $N_{s, \varphi}^{+}=N^{+}$. 
Now it is easy to complete the proof of Theorem 1 . The range of the operator (6) coincides with the set (7) because the range is closed and $N^{+}$ is the kernel of the adjoint to (6) (with respect to the inner product in $\left.\left(L_{2}(\Gamma, d x)\right)^{p}\right)$. The index of $(6)$ is equal to $\operatorname{dim} N-\operatorname{dim} N^{+}$as the spaces $\left(H^{s, \varphi}(\Gamma)\right)^{p} / A\left(H^{s, \varphi}(\Gamma)\right)^{p}$ and $N^{+}$have the same dimension.

Theorem 1 is proved.

Proof of Theorem 2. This theorem follows from the first equality of (12). Indeed, let $s \in \mathbb{R}, \varphi \in \mathcal{M}$ and $\sigma>0$. Denote by $\|\cdot\|_{s, \varphi}^{\prime},\|\cdot\|_{s, \varphi}^{\prime \prime}$ and $\|\cdot\|_{s-\sigma, \varphi}^{\prime}$ the norms in

$$
\prod_{k=1}^{p} H^{s+m_{k}, \varphi}(\Gamma), \quad\left(H^{s, \varphi}(\Gamma)\right)^{p} \text { and } \prod_{k=1}^{p} H^{s+m_{k}-\sigma, \varphi}(\Gamma)
$$

respectively. For any $u \in\left(\mathcal{D}^{\prime}(\Gamma)\right)^{p}$ and $f=A u$ we have

$$
\|u\|_{s, \varphi}^{\prime}=\left\|B f-T_{1} u\right\|_{s, \varphi}^{\prime} \leq\|B f\|_{s, \varphi}^{\prime}+\left\|T_{1} u\right\|_{s, \varphi}^{\prime} \leq c_{0}\|f\|_{s, \varphi}^{\prime \prime}+c_{1}\|u\|_{s-\sigma, \varphi}^{\prime} .
$$

Here, $c_{0}$ is the norm of the bounded operator (13), and $c_{1}$ is the norm of the operator

$$
T_{1}: \prod_{k=1}^{p} H^{s+m_{k}-\sigma, \varphi}(\Gamma) \rightarrow \prod_{k=1}^{p} H^{s+m_{k}, \varphi}(\Gamma) .
$$

The last operator is bounded by Lemma 1 because all elements of the matrix $T_{1}$ belong to $\Psi^{-\infty}(\Gamma)$. Inequality (16) is equivalent to Theorem 2 (we use non-Hilbertian norms in this theorem to avoid an awkward formula).

Finally, Theorem 3 is a direct consequence of Theorem 1.

6. An application. Let $\Gamma_{0}$ be an open nonempty subset of the manifold $\Gamma$. We define

$$
H_{\mathrm{loc}}^{s, \varphi}\left(\Gamma_{0}\right):=\left\{f \in \mathcal{D}^{\prime}(\Gamma): \chi f \in H^{s, \varphi}(\Gamma) \forall \chi \in C^{\infty}(\Gamma) \text {, supp } \chi \subseteq \Gamma_{0}\right\} .
$$

Theorem 4. Assume that $u \in\left(\mathcal{D}^{\prime}(\Gamma)\right)^{p}$ is a solution of the equation $A u=f$ on $\Gamma_{0}$, where $f \in\left(H_{\text {loc }}^{s, \varphi}\left(\Gamma_{0}\right)\right)^{p}$ for some $s \in \mathbb{R}$ and $\varphi \in \mathcal{M}$. Then $u \in \prod_{k=1}^{p} H_{\mathrm{loc}}^{s+m_{k}, \varphi}\left(\Gamma_{0}\right)$.

This theorem specifies, with regard to the refined scale (4), the known propositions on local increase of interior smoothness of an elliptic system solution in the Sobolev scale (see [5, 9, 4]). Note that the refined local smoothness $\varphi$ of the right-hand side of the elliptic system is inherited by its solution.

First we prove Theorem 4 in the global case where $\Gamma_{0}=\Gamma$.

Lemma 2. Let $s \in \mathbb{R}, \varphi \in \mathcal{M}$ and $u \in\left(\mathcal{D}^{\prime}(\Gamma)\right)^{p}$. Then

$$
A u \in\left(H^{s, \varphi}(\Gamma)\right)^{p} \Rightarrow u \in \prod_{k=1}^{p} H^{s+m_{k}, \varphi}(\Gamma) .
$$


Proof. Assume that $A u \in\left(H^{s, \varphi}(\Gamma)\right)^{p}$. In view of (12), (13) we have

$$
u=B A u-T_{1} u, \quad B A u \in \prod_{k=1}^{p} H^{s+m_{k}, \varphi}(\Gamma) .
$$

In addition, since all elements of the matrix $T_{1}$ are in $\Psi^{-\infty}(\Gamma)$, we have $T_{1} u \in\left(C^{\infty}(\Gamma)\right)^{p}$ (see [1, Sec. 2.1]). Lemma 2 is proved.

Proof of Theorem 4. Let us show that the condition $f \in\left(H_{\mathrm{loc}}^{s, \varphi}\left(\Gamma_{0}\right)\right)^{p}$ implies the following local smoothness increase of the solution $u$ : for every $r \geq 1$,

$$
u \in \prod_{k=1}^{p} H_{\mathrm{loc}}^{s-r+m_{k}, \varphi}\left(\Gamma_{0}\right) \Rightarrow u \in \prod_{k=1}^{p} H_{\mathrm{loc}}^{s-r+1+m_{k}, \varphi}\left(\Gamma_{0}\right) .
$$

Choose $\chi, \eta \in C^{\infty}(\Gamma)$ such that $\operatorname{supp} \chi$, supp $\eta \subseteq \Gamma_{0}$ and $\eta=1$ in a neighborhood of supp $\chi$. Interchanging the matrix pseudodifferential operator $A$ and the operator of multiplication by $\chi$ we can write

$$
\begin{aligned}
A \chi u & =A \chi \eta u=\chi A \eta u+A^{\prime} \eta u=\chi A u+\chi A(\eta-1) u+A^{\prime} \eta u \\
& =\chi f+\chi A(\eta-1) u+A^{\prime} \eta u \quad \text { on } \Gamma .
\end{aligned}
$$

Here, the matrix pseudodifferential operator $A^{\prime}=\left(A_{j, k}^{\prime}\right)_{j, k=1}^{p}$ is the commutator of these operators. Since $A_{j, k}^{\prime} \in \Psi^{m_{k}-1}(\Gamma)$, there exists (by Lemma 1) the bounded operator

Therefore

$$
A^{\prime}: \prod_{k=1}^{p} H^{s-r+m_{k}, \varphi}(\Gamma) \rightarrow\left(H^{s-r+1, \varphi}(\Gamma)\right)^{p} .
$$

$$
u \in \prod_{k=1}^{p} H_{\mathrm{loc}}^{s-r+m_{k}, \varphi}\left(\Gamma_{0}\right) \Rightarrow A^{\prime} \eta u \in\left(H^{s-r+1, \varphi}(\Gamma)\right)^{p} .
$$

Further, since $f \in\left(H_{\mathrm{loc}}^{s, \varphi}\left(\Gamma_{0}\right)\right)^{p}$, in view of Proposition 1(iv) we have

$$
\chi f \in\left(H^{s, \varphi}(\Gamma)\right)^{p} \hookrightarrow\left(H^{s-r+1, \varphi}(\Gamma)\right)^{p} .
$$

In addition, since the supports of the functions $\chi$ and $\eta-1$ are disjoint,

$$
\chi A(\eta-1) u \in\left(C^{\infty}(\Gamma)\right)^{p} .
$$

It follows from (18)-(21) that

$$
u \in \prod_{k=1}^{p} H_{\mathrm{loc}}^{s-r+m_{k}, \varphi}\left(\Gamma_{0}\right) \Rightarrow A \chi u \in\left(H^{s-r+1, \varphi}(\Gamma)\right)^{p} .
$$

Next, according to Lemma 1 we have

$$
A \chi u \in\left(H^{s-r+1, \varphi}(\Gamma)\right)^{p} \Rightarrow \chi u \in \prod_{k=1}^{p} H^{s-r+1+m_{k}, \varphi}(\Gamma) .
$$


The implication (17) is a consequence of the last two implications because $\chi \in C^{\infty}(\Gamma)$ with supp $\chi \subseteq \Gamma_{0}$ is arbitrary.

Now it is easy to deduce Theorem 4 from the implication (17). Since $\Gamma$ is compact, $\mathcal{D}^{\prime}(\Gamma)$ is the union of the Sobolev spaces $H^{\sigma}(\Gamma), \sigma \in \mathbb{R}$. So, there is a sufficiently large integer $r_{0}>0$ such that

$$
u \in \prod_{k=1}^{p} H^{s-r_{0}+1+m_{k}}(\Gamma) \subset \prod_{k=1}^{p} H_{\mathrm{loc}}^{s-r_{0}+m_{k}, \varphi}\left(\Gamma_{0}\right) .
$$

From this by applying (17) for $r=r_{0}, r_{0}-1, \ldots, 1$ in succession, we deduce Theorem 4:

$$
\begin{aligned}
u \in \prod_{k=1}^{p} H_{\mathrm{loc}}^{s-r_{0}+m_{k}, \varphi}\left(\Gamma_{0}\right) \Rightarrow u \in \prod_{k=1}^{p} H_{\mathrm{loc}}^{s-r_{0}+1+m_{k}, \varphi}\left(\Gamma_{0}\right) \\
\Rightarrow \cdots \Rightarrow u \in \prod_{k=1}^{p} H_{\mathrm{loc}}^{s+m_{k}, \varphi}\left(\Gamma_{0}\right) .
\end{aligned}
$$

Theorem 4 and Proposition 3 imply the following sufficient condition for a fixed component $u_{k}$ of the solution of (2) to have continuous derivatives of a prescribed order.

TheOREM 5. Suppose that $u, f \in\left(\mathcal{D}^{\prime}(\Gamma)\right)^{p}$ satisfy the equation $A u=f$ on $\Gamma_{0}$. Let $\varrho \geq 0$ and $k=1, \ldots, p$ be integers, and let $\varphi \in \mathcal{M}$ satisfy the inequality (5). Then

$$
\left(f_{j} \in H_{\mathrm{loc}}^{\varrho-m_{k}+n / 2, \varphi}\left(\Gamma_{0}\right), j=1, \ldots, p\right) \Rightarrow u_{k} \in C^{\varrho}\left(\Gamma_{0}\right) .
$$

\section{References}

[1] M. S. Agranovich, Partial Differential Equations. VI. Elliptic Operators on Closed Manifolds, Encyclopaedia Math. Sci. 63, Springer, Berlin, 1994, 1-130.

[2] Sh. A. Alimov, V. A. Il'in and E. M. Nikishin, Convergence problems of multiple trigonometric series and spectral decompositions. I, Russian Math. Surveys 31 (1976), no. 6, 29-86.

[3] M. F. Atiyah and I. M. Singer, The index of elliptic operators on compact manifolds, Bull. Amer. Math. Soc. 69 (1963), 422-433.

[4] Yu. M. Berezanskij, Expansions in Eigenfunctions of Selfadjoint Operators, Transl. Math. Monogr. 17, Amer. Math. Soc., Providence, 1968.

[5] A. Douglis and L. Nirenberg, Interior estimates for elliptic systems of partial differential equations, Comm. Pure Appl. Math. 8 (1955), 503-538.

[6] W. Farkas and H.-G. Leopold, Characterisations of function spaces of generalized smoothness, Ann. Mat. Pura Appl. 185 (2006), 1-62.

[7] C. Foiaş et J.-L. Lions, Sur certains théorèmes d'interpolation. Acta Sci. Math. (Szeged) 22 (1961), 269-282.

[8] D. D. Haroske and S. D. Moura, Continuity envelopes of spaces of generalised smoothness, entropy and approximation numbers, J. Approx. Theory 128 (2004), 151-174. 
[9] L. Hörmander, Linear Partial Differential Operators, Springer, Berlin, 1963.

[10] - The Analysis of Linear Partial Differential Operators. II. Differential Operators with Constant Coefficients, Springer, Berlin, 1983.

[11] —, The Analysis of Linear Partial Differential Operators. III. Pseudo-Differential Operators, Springer, Berlin, 1985.

[12] G. A. Kalyabin and P. I. Lizorkin, Spaces of functions of generalized smoothness, Math. Nachr. 133 (1987), 7-32.

[13] V. A. Mikhailets, Asymptotics of the spectrum of elliptic operators and boundary conditions, Soviet Math. Dokl. 26 (1982), 464-468.

[14] - A precise estimate of the remainder in the spectral asymptotics of general elliptic boundary problems, Funct. Anal. Appl. 23 (1989), 137-139.

[15] V. A. Mikhailets and A. A. Murach, Improved scale of spaces and elliptic boundaryvalue problems. I, Ukrainian Math. J. 58 (2006), 244-262.

[16] - , - Improved scale of spaces and elliptic boundary-value problems. II, ibid., 398-417.

[17] —, -, Regular elliptic boundary-value problem for a homogeneous equation in a two-sided refined scale of spaces, ibid., 1748-1767.

[18] -, - Elliptic operator with homogeneous regular boundary conditions in two-sided refined scale of spaces, Ukrainian Math. Bull. 3 (2006), 529-560.

[19] - - - Refined scale of spaces and elliptic boundary-value problems. III, Ukrainian Math. J. 59 (2007), 744-765.

[20] - , - An elliptic boundary-value problem in a two-sided refined scale of spaces, Ukrain. Mat. Zh. 60 (2008), 497-520 (in Russian).

[21] - , - Interpolation with a function parameter and refined scale of spaces, Methods Funct. Anal. Topology 14 (2008), 81-100.

[22] A. A. Murach, Elliptic pseudo-differential operators in a refined scale of spaces on a closed manifold, Ukrainian Math. J. 59 (2007), 874-893.

[23] B. Paneah, The Oblique Derivative Problem. The Poincaré Problem, Wiley - VCH, Berlin, 2000.

[24] G. Slenzak, Ellptic problems in a refined scale of spaces, Moscow Univ. Math. Bull. 29 (1974), no. 3-4, 80-88.

[25] L. R. Volevich and B. P. Paneah, Certain spaces of generalized functions and imbedding theorems, Uspekhi Mat. Nauk 20 (1965), no. 1, 3-74 (in Russian).

Vladimir A. Mikhailets

Institute of Mathematics NAS of Ukraine

Tereshchenkivska St. 3

Kyiv 01601, Ukraine

E-mail: mikhailets@imath.kiev.ua
Aleksandr A. Murach Institute of Mathematics NAS of Ukraine

Tereshchenkivska St. 3 Kyiv 01601, Ukraine and

Chernigiv State Technological University

Shevchenka St. 95

Chernigiv 14027, Ukraine E-mail: murach@imath.kiev.ua 